\title{
A Study of the Client Culture Influences on the Creation of Tailored Design Homes in New Communities Around Cairo
}

\author{
Karim Kesseiba, \\ Cairo University
}

\begin{abstract}
The design process of residential homes is one of the most dynamic ones, due to its strong interrelation with the life patterns, economical strategies, needs and cultural traits of the clients. This is relevant in the case of tailored design homes in the new suburbs around Cairo, where the client aims to fulfill several of his life goals and visions, in addition to the possible freedom of design creations which are not as much bounded to the limitations inside the city. Accordingly, the architect has to deal with twofold aspects, first is aiming to understand and react to the clients culture and second to produce accepted architectural values and qualities. Although both aspects appear to be intertwined, however, case studies reflected that in several aspects, the clients' culture affects the production of the aspired design vision. Thus, the paper aims to discuss the dilemma through two case studies in one of the gated communities around Cairo, based on qualitative analysis of how the designer managed to mediate the design of tailored homes between the clients' culture and the production of valued architectural quality. This study will be based on the brief and anonymous explanation of the social profile of the clients' social background which acted as the motivator in the design process, based on interviews with architect who worked in the design development process.
\end{abstract}

Keywords: culture, domestic tailored design, design process, design patterns.

\section{Introduction}

The design process of residential houses is one of the most dynamic ones, due to its strong interrelation with life patterns, economical strategies, needs and cultural background of the clients. This is relevant in the case of tailored design homes in the new suburbs around Cairo, where the client aims to fulfill their dream homes in addition to the possible freedom of design facilities which are not as much bounded to the limitations inside the condensed city context. Accordingly, the architect has to deal with twofold aspects, first is aiming to understand and react to the clients culture and second to produce accepted architectural values and qualities. Although both aspects appear to be intertwined, however, case studies reflected that in several aspects, the clients' culture affects the production of the design based on their personal traits. Thus, the paper aims to discuss the dilemma through two case studies in one of the gated communities around Cairo, based on qualitative analysis of how the designer managed to mediate the design of tailored homes between the clients' culture and the production of valued architectural quality. This study will be based on the brief and anonymous explanation of the social profile of the clients' social background which acted as the motivator in the design process, based on interviews with architects who worked in the design development process. In addition to this, analysis of the design outcome interrelated with the clients' intervention will be analyzed. To better understand this analysis, a brief literature review will be made to critique and highlight the inter-relation between culture and the materialization of behavioral patterns in domestic architecture.

\section{Literature Review and Critique of the inter-relation between Culture and Domestic Architecture Design}

This part is concerned with the critique of how culture affects the design patterns and outcomes of dwellings and homes. The statement of "an Englishman's home is his castle" reflects that while individuals and families might face a lifelong struggle for self assurance in the public life, "the private home is a 'haven' or 'retreat' where we are free to express our individualism in whatever way we choose", (Chapman and Hockey, 2002). In accordance with that, Chapman and Hockey, (2002) further emphasize that the 'home' as an idea, place and object consumes a large proportion of individuals' incomes, and penetrates their dreams and their leisure time while the physical absence of a home presents a major problem to both 
society and the homeless themselves. And as a theoretical relevance, it is agreed upon that consistency of popular images and experiences of home life can produce vital clues as to how society's members both produce and respond to social change, (Chapman and Hockey, 2002). This is further asserted by the fact that the alterations in the home's designs come about in response to changed patterns of urban life, employment, expectations of leisure, privacy, respectability, community, security and the projection of social status. Those aspects will be very important to reflect upon in the analysis to regard how new homes in Cairo suburbs can be a translation for those cultural aspects.

Raporport (1985) further exposes that "home environments comprise most of the built environment. They also have extraordinarily high affective (and often economic and social) significance: they are the primary settings par excellence". That's why they are of great interest to users. They are also of great interest to researchers. From a social and communal perspective, Raporport (1985) argue that if homes are not chosen and designed they are not conceptually "home". An imposed setting is unlikely to be a home environment.

This critique forces the designer of "homes" rather than houses to question the following aspects before the design is created, (Rapporport, 1985), what characteristics of people, as members of a group, or as individuals, influence (or, in design, should influence) how built environments are shaped? What are the effects of the built environment on human behavior, well-being, mood, and so forth? What mechanisms link people and environments in this mutual interaction? Those kinds of questions will be further exposed in the analysis of dwellings according to social profiles and activity patterns in the upcoming part of the research.

It is important to note that there are several constraints which obstruct the creation of pure "dream homes" in the physical settings. Those constraints as Raporport (1985) lists are the resource constraints, what can be afforded, what is in fact, available to the individual or group, and the market constraints of supply and price. Accordingly, the analysis will focus on cases of similar constraints to limit the variables to cultural traits.

Furthermore, home environments are part of larger, culturally variable systems of settings and are themselves best understood as that system of settings within which a particular set of activities takes place disregarding the variability of activities and, specially, their latent aspects. For example, the type of work and where and when it occurs influences the choice of home environment. Should work become more home centered as many predict the nature of home environments will also change. This is evident in the different settings of houses and their design patterns across history which will be discussed below.

From another side, different groups may see home environments differently: primarily as settings for family life, as indications of prestige or status, or as safe havens. Each emphasis leads to a different environmental quality profile. Different profiles are associated with changes in use, for example, housing as a setting for private recreation or as a workplace. Profiles can help interpret studies of preference for residential environments, although group variability and associational considerations must be included, (Raporport, 1985).

Through a brief historical review of how social patterns affect the design patterns of homes, Chapman and Hockey (2002), show that the open-plan design of 1960s housing removes that private space within which women formerly managed tasks such as cooking, sewing, washing and ironing. In the absence of separate kitchens, outhouses, dining rooms and parlours, women experience an additional pressure to maintain the entire ground floor of their homes in a state of order equivalent to that once required only in the secluded space of the front room. As Le Corbusier sets out objectively, the house is a "machine for living". He envisioned a desire to produce works of art and design based on objectivity and rationality. Based on that, facing social and economic problems after World War II, and for the purpose of solving complex design problems and meeting user requirements, the fact of design was considered as a problem-solving and decision-making activity, (Bayazit, 2004).

In addition to this, as Walker (2002) explains that modernist architects, most importantly Le Corbusier, Mies van der Rohe, and Walter Gropius, strongly rejected the homey values, national character, and decorative aesthetic of the Victorian period and defined modernism against domesticity. The modern home was reconceived as a machine like stated previously. Modernist architectural language was itself "objective," stripped of any lingering Victorian sentimentality or domesticity. Like the words that architects used to describe them, modernist houses, such as Le Corbusier's canonical Villa Savoye of 1929, near Paris (fig. 1), were also stripped of ornament and domesticity. In the so-called new architecture, decoration, traditionally associated with the feminine, became a hallmark of bad design. Although purportedly gender neutral and 
universalist in its values, modernist domestic space was implicitly masculine, defining, and controlling, operating through surveillance and the "domination of the gaze", Walker (2002).

Walker (2002) further presents that the modernist approach that proposed the architect as a manipulator of seemingly neutral, abstract space also pursued the "home as laboratory," a site of social and formal experimentation that failed most significantly and visibly in the large-scale, socially disastrous superblocks of postwar public housing but succeeded most fully in tailored private houses, such as the Eames House in California (1949-50).In postmodern culture, architectural approaches that link theory to practice have also flourished within the conditions of urban domesticity.

\section{Critical Analysis of How Clients' Culture Impacts the Design Product for Tailored Design Homes: Case Study Analysis}

People spend more time in their homes than in any other space. The home ideally provides a safe, comfortable environment in which to relax, communicate, learn, and be entertained. Increasingly, it is where people connect with friends and family, conduct business, manage resources, learn about the world, and maintain health and autonomy as they age. People invest considerable amounts of time, money, and emotional energy to mold their homes into living spaces that meet their needs.

It is crucially important to regard the value and importance of enhancing architectural design for the homes according to the debates discussed above and also stemming from what Hillier et al (1987) imply that "architectural design affects human behavior in some way that it acts as an independent variable in a describable process of cause and effect". In addition to this, "domestic space layout reflects cultural codes in which activity patterns, styles and spatial layouts all seem to be interrelated", (Hillier et al, 1987).

This approach connects the debates to another scale of adapting the patterns, activities and needs of the users in blended design criteria to balance the needs Vs. the architectural quality. As Nigel (1982) presents, design is a process of pattern "synthesis", rather than "pattern recognition". The solution is not simply lying there among the data; it has to be actively constructed by the designer's own efforts. Thus, the main role of the designer is to use such a code, which enables the designer to effect a translation from individual, organizational and social needs to physical artifacts. This code is supposed to express and contain actual connections which exist between human needs and their artificial environment. In effect, the designer learns to 'speak' a language, to make a useful transaction between domains which are unlike each other (sounds and meanings in language, artifacts and needs in design) by means of a code or system of codes which structure that connection, Nigel (1982).

From the personal level of the individual client, Gautam et al (2007) define culture as a sort of 'software of the mind'. Accordingly, every person carries patterned ways of thinking, feeling and reacting. These are partly unique and partly shared with others. The unique part belongs to the individual's personality level. The common part which belongs to the collective level taking into account that culture is a collective phenomenon and is shared at least partially with other people living in the same social group, either on the level of the family, or the group membership on the social class levels or the national levels. Thus, culture can be treated as the collective programming of the mind that distinguishes the members of one group or category of people from another.

The next part will address the case studies selected for analysis based on the previously exposed debates of how the users' culture, profession and background affects the creation of the spaces inside the home and the inside-out spatial relation. The two cases represent two different approaches for dealing with the spaces however, two main variables were not changed to reflect upon the possibility of creating totally different approaches will maintaining the profession of the users. In addition to this, both houses are located in Cairo's suburb, 6th of October, and were custom-made homes not modeled houses. The analysis which is based on the architect's interview will cover three main points, first the social profile, second the spatial organization of the main living space and third is the relation between the inside and the outside spaces.

\subsection{Case Study One: The Home of an Architect}

This case study chosen for analysis here will represent the values of aesthetics created by an architect who designed his own home for his family, with an annexed studio and a guest suite. The analysis will cover the previously stated points, the social profile, the inside-out relation and the concept of the inter-relation between the main living room space and the hobby spaces. 


\subsubsection{Social Profile}

The home accommodates a family of four members and possible visits from parents. The social profile of the users are the architect who is also a professor and needs space for the work meetings or design production inside the home, however, without a compromise of the privacy of the spaces for his wife and two daughter, who mainly do most of the activities in the living room and the outside garden and swimming pool. The architect also needed a space for his hobby, playing squash inside the home. In addition to this, a separate however linked to the home guest suite was required for the parents who pay short visits.

This profile affected the design of the home through several main points, first the need to create an annexed studio space within the home complex which has access from the outside and interesting views, without compromising the private spaces of the rest of the family members. This was solved architecturally via the space shown below, (fig. 2 ) in which the studio is linked as a separate mass to the outside entrance and the main entrance of the home. To achieve a more valuable addition of the annexed the studio, the roof was utilized for photovoltaic panels, which add a sustainable dimension to the design.

The other main players which affected the design were the possibility of receiving guests as parents of the architect, whose guest suite was preferred to be located within the same mass as the home, visually linked to the main recreational spaces, however, maintaining their privacy. Thus, a studio was added on the second floor as shown below (fig. 3), accommodating the living room, the workspace of the parents, and the private kitchen all as one open plan design, with a private entrance from the main lobby space of the rest of the home.

\subsubsection{Inside-out relations}

The main concept behind the design of the inside-out relation as presented in the architect's sketches displayed below and the outcome of the realistic design, (figs. 4-7) is to create a dynamic link to the outside environment through several aspects. First the creation of extended terraces linking the main reception area, the living room and the guest suite with the outside pool area and garden. From another side, maintain the maximum privacy of the living room which is this case is located in the basement level, in direct contact visually and functionally with the pool but not compromising exposure.

\subsubsection{Main Living spaces and hobby spaces}

The heart of the home, or the main living and hobby spaces are linked internally as shown below (figs. 8-11). The design was targeting the maximum utilization of the functional and energetic space while maintain the conservative background of the users and the aim to create privacy in the shadows of the dynamic use of the household. Thus, as shown below, the basement level links the living room to the everyday kitchen of the home all in one open plan setting. The privacy was maintained by the extension of the upper terraces, as well as the segregation of the reception and studios in different levels.

\subsection{Case Study Two: The Home of a Newly-wed}

This case study embodies a different approach of how architects deal from a spatial organization aspect with the varying need of clients. The case study exposed below is a home tailored for the needs of a newly married couple whose social profile will be exposed below to analyze how this affected the design.

\subsubsection{Social Profile}

The users whom the architect dealt with in this case were both fresh graduates who adopted a more introvert approach of creating all the needs of the home and hobbies, such as sketching and reading inside the home with lesser interaction with the outside environment. There were no frequent visitors expected thus there was no need to create a guest space. Also both worked independently outside the home, so merely a space for home activities was required not a formal meeting space with outsiders.

\subsubsection{Inside-Out Relations}

Based on the social profile explained above, the inside space was created as a triple height vertical open space linking the heart of the home with the livable spaces. This was more or less a re-interpretation of the inner courtyard of old homes, yet, through a contemporary encounter. It was appreciated by the users to limit the horizontal extensions via open terraces, however, on the contrary to create several openings to enhance natural light, shades and shadows in the inner spaces. In addition to this, it was required to create extended walls for hanging photos or sketches and books. Since all the spaces 
are introvert space, it was essential for the architect to create a dynamic inner space to provide an aesthetic experience in the inside. This was created as shown below (figs. 12-14) by maintaining the inner heart of the home visually and spatially linked to the main pitched roof experience of the home.

\subsubsection{Main Living Space}

The main living space of the home was in need to be linked to the workspace visually as shown below (figs. 15, 16). This was enhanced through an open plan strategy adopted by the designer, to interrelate the horizontal connectivity of the spaces with the vertical visual connectivity. From another side as was shown in the figures above, the open plan of the first floor connected the daily pantry space with the living room to maintain a utilitarian flexibility for the users.

\section{Discussion}

Based on the previously studied literature review and the analysis conducted on the two case studies maintaining the same variables of being custom-made homes in Cairo's suburbs, great variation can be tackled here as to the influence of the client's needs and cultural traits on the design of the homes. Primarily are the horizontal and vertical settings of the homes, based on the life patterns and activities of the user. The first case in which the home accommodates complicated hierarchies of users each with different requirements and activities provided a challenge for the architect to create a dynamic complex which inhibits several spaces visually connected as one home yet totally varying in treatments in the scale of the horizontal connectivity.

From another side, the second case study reflected a lesser complexity of uses; however, the main challenge for the architect was to create a dynamic inner space which aids in the patterns, lifestyle and activities of the users. This was maintained through the introvert yet flexible and dynamic horizontal attachments of spaces.

\section{Conclusion}

The paper aimed to highlight a very important dilemma in the contemporary expansions of suburbs which is the personal variations of client's needs and culture in their home $V s$ the canned houses created by the real estate developers in which the user is merely offered models to choose from without any tailoring to the specific needs of the client in this category of homes.

The literature review highlighted that what we are suffering now in Cairo's expansions to the East and to the West was more or less similar to the European dilemma post World War II, reacting in the prefabricated canned houses. However, it is clear that personal differences of clients create richer built environments and provide an opportunity for limitation of the chaotic reactions in the built models seen in several gated compounds around Cairo. All this can be created within the already provided economics, however, merely providing quality of the built product.

\section{References}

[1] Bayazit, N. (2004) "Investigating Design: A Review of Forty Years of Design Research", Design Issues: vol. 20, no. 1, Massachusetts Institute of Technology.

[2] Chapman, T. and Hockey, J., (2002). "IDEAL HOMES? Social change and domestic life", Routledge.

[3] Gautam, V. et al. (2007), "Cultural Influences On The Design Process", International Conference On Engineering Design, Iced'07.

[4] Hillier, B. et al (1987), "Creating Life or Does Architecture Determine Anything?", Arch. And Comport., Arch Behaviour, Vol. 3, no. 3.

[5] Nigel, C. (1982). "Designerly ways of knowing". Design Studies, 3(4) pp. 221-227.

[6] Rapoport , A.,(1985). "Thinking about Home Environments, A Conceptual Framework", in Altman I. et al. (eds.), Home Environments, Springer Science+ Business Media, New York.

[7] Walker, L. (2002), "Home Making: An Architectural Perspective", Signs: Journal of Women in Culture and Society, vol. 27, no. 3, The University of Chicago. 


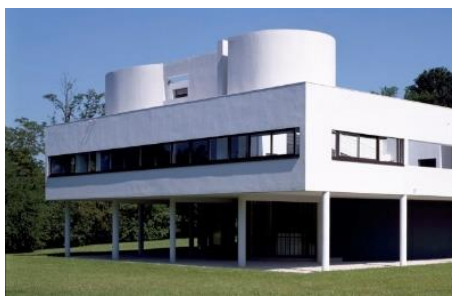

Figure 1, Le Corbuseir's Villa Savoy, https://www.khanacademy.org/humanities/apart-history/later-europe-andamericas/modernity-ap/a/corbusier-savoye, 2017.

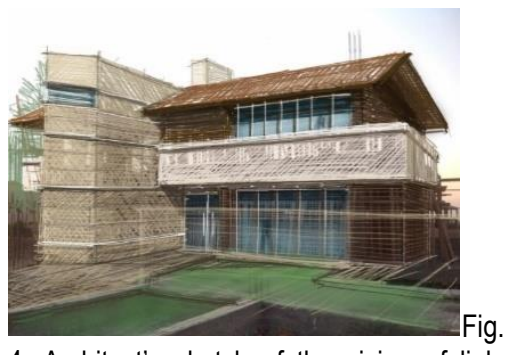

4, Architect's sketch of the vision of linked terraces, Author, 2017

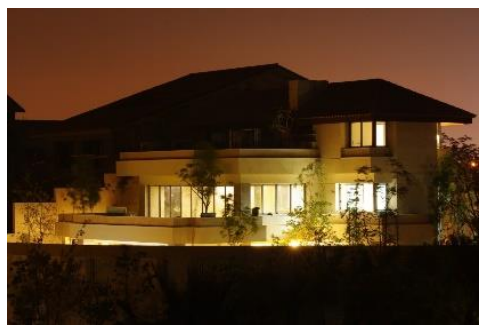

Fig. 7, Extended Terraces linking outside spaces with the reception and guest house, Author, 2017.

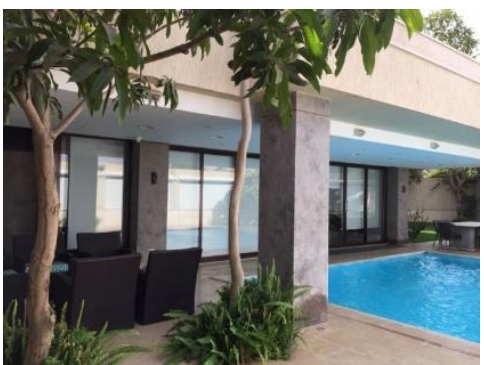

Figs. 10-11, Living room space connected with the kitchen in a direct open plan setting with the pool level, Author, 2017
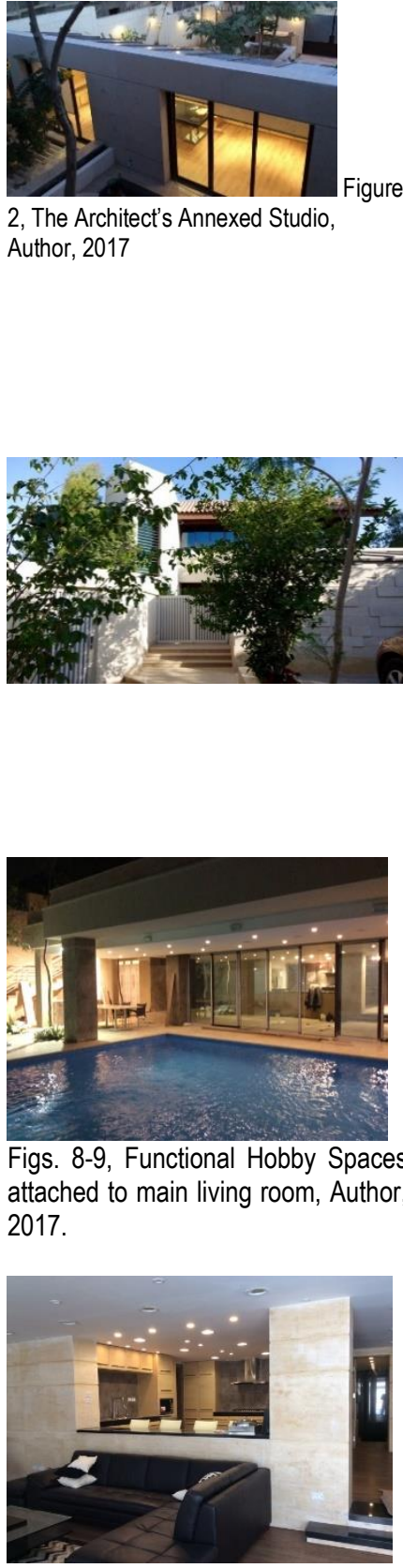

2, The Architect's Annexed Studio, Author, 2017
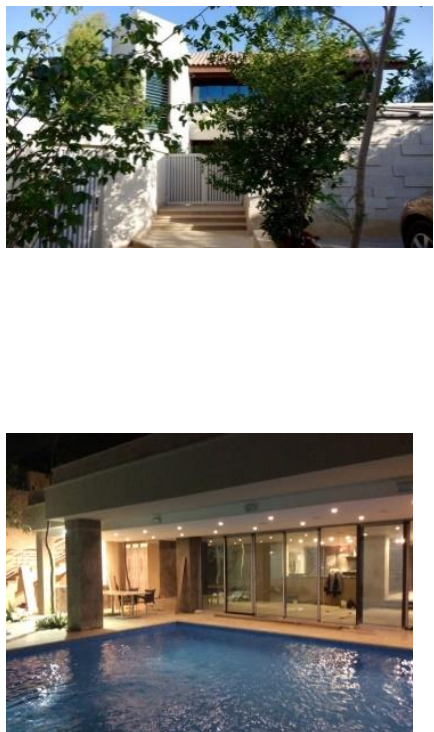

Figs. 8-9, Functional Hobby Spaces attached to main living room, Author, 2017.
Fig. 12, Main vertical inner space linking the main spaces of the home, Author, 2017

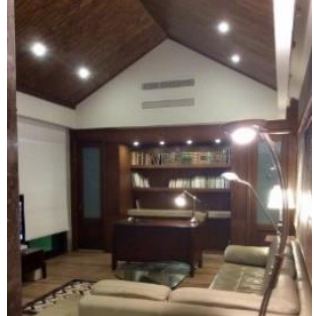

Figure 3, The Architect's Parents Guest Suite Located in the last floor, Author, 2017

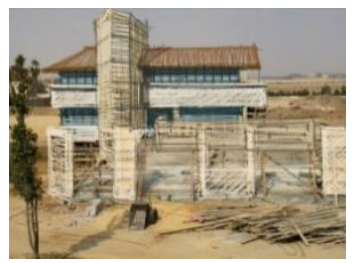

Fig. 5-6, Architect's sketches and vision Vs achieved building, Author, 2017.
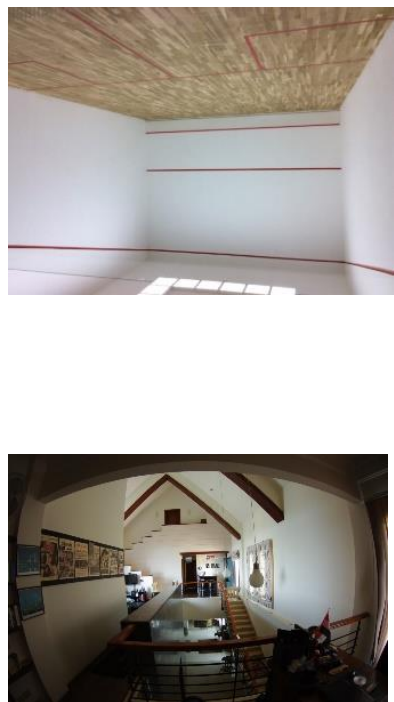

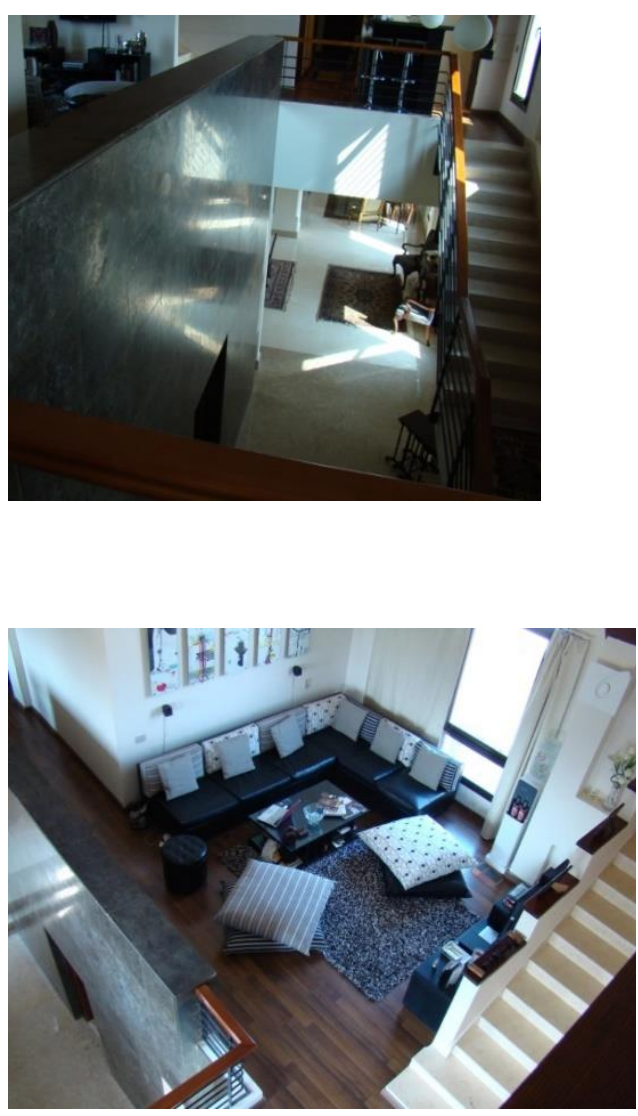

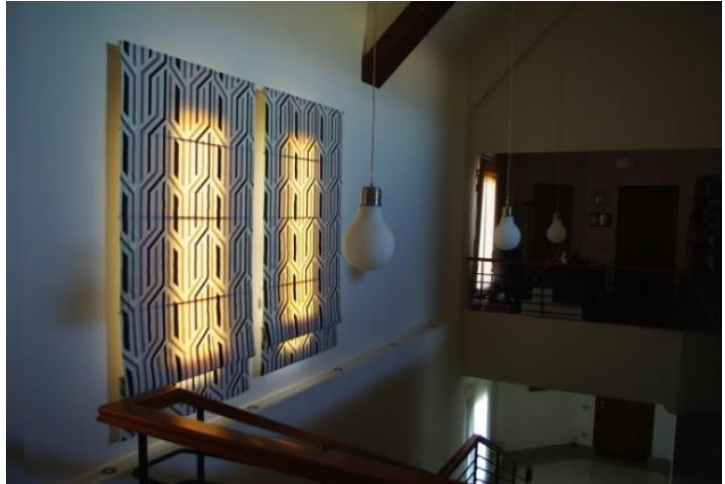

Figs. 13-14, Main vertical connections linking visually the different workspaces, the living room and the open space, Author, 2017.

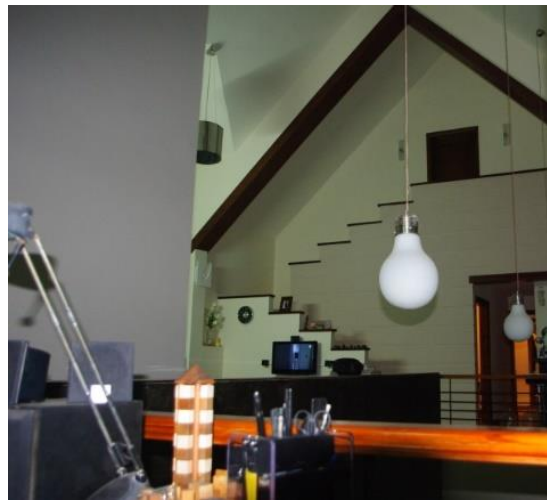

Figs. 15-16, The living room acting as the heart of the home horizontally connected to the workspace and vertically connected the open inner space, Author, 2017. 